\title{
Orbital cellulitis
}

\author{
D. P. MARTIN-HIRSCH, S. HABASHI, A. H. HINTON \\ \& B. KOTECHA \\ University Department of ENT Surgery, Manchester Royal Infirmary, Manchester
}

\section{SUMMARY}

Orbital cellulitis is an emergency. It may cause blindness and progress to lifethreatening sequelae such as brain abscess, meningitis and cavernous sinus thrombosis. Successful management is dependent upon urgent referral and immediate treatment. Although isolated eyelid erythema and swelling usually indicate primary infection anterior to the orbital septum, they may also be the first signs of an underlying frontal or ethmoidal sinusitis. The condition always requires emergency referral to both an opthalmologist and otorhinolaryngologist.

\section{INTRODUCTION}

The aim of this paper is to alert clinicians to the signs, symptoms, aetiology and management of orbital cellulitis. Three cases are reported to highlight the importance of early diagnosis and adequate treatment.

\section{CASE REPORT 1}

A 50-year-old woman presented to the Accident and Emergency Department with a 2-week history of mucopurulent rhinorrhoea and a 48-h history of progressive upper eyelid swelling and tenderness.

On examination the patient was febrile with gross localized swelling of the upper eyelids. (Figs $1 \& 2$ ) Her conjuctivae were injected. There was no evidence of proptosis or limitation of eye movement. Visual acuity was normal.

The patient had a raised white cell count and sinus $\mathrm{X}$-rays revealed pansinusits. 


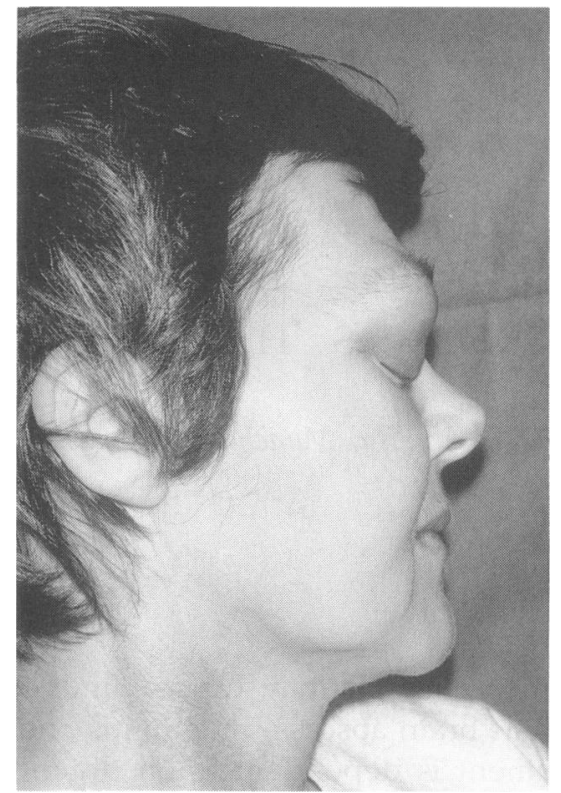

Fig. 1. Profile of patient showing gross localized swelling.

Blood was drawn for culture and i.v. antibiotics administered. Antral washouts were carried out.

With no improvement in the patient's condition after twelve hours ethmoidectomy and drainage of bilateral pre-septal abscesses were performed. The patient made a good recovery and was discharged three days later without visual deficit.

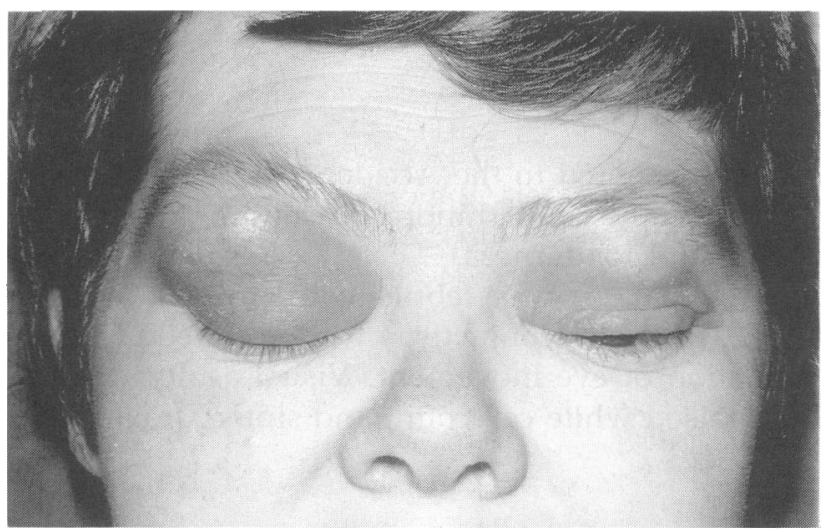

Fig. 2. Gross localized swelling of upper eyelids 


\section{CASE REPORT 2}

A 43-year-old fireman presented to the Accident and Emergency Department with a 3-week history of purulent rhinnorhoea, throbbing facial pain and a 2-day history of progressive right eye pain and swelling, which had been treated by his GP with oral antibiotics.

On examination the eye was proptosed, eye movements were painful and restricted and visual acuity was diminished. Anterior rhinoscopy revealed bilateral nasal polyps and pus.

An urgent C.T. Scan demonstrated a pansinusitis with erosion of the medial bony wall of the right orbit. There was gross periorbital swelling with early retroorbital abscess formation (Fig. 3). Urgent right fronto-ethmoidectomy and intranasal antrostomies revealed extensive polypoidal disease.

The patient was discharged 1-week later with residual diminished visual acuity. He subsequently lost his job as a fireman.

\section{CASE REPORT 3}

A 54-year-old woman sustained a fracture of the right maxilla and ethmoid complex and was seen at her local Accident Emergency Department. She was discharged without antibiotic cover.

Five days later the patient re-presented. On examination there was gross right proptosis with surrounding cellulitis, opthalmoplegia and no clinically demonstrable vision. The patient was febrile and toxic.

Urgent CT Scan showed fracture of the anterior wall of the right maxillary

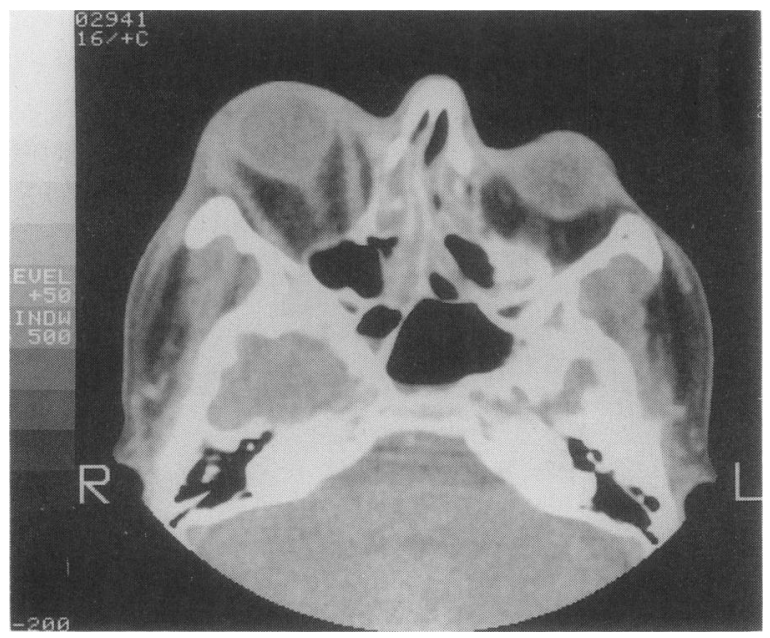

Fig. 3. C.T. scan showing a pansinusitis with erosion of the bony wall of the right orbit and gross peri-orbital swelling with early retro-orbital abscess formation. 
antrum, right ethmoidal air cell complex and the anterior margin of the orbital floor. There was extensive enhancing peri-orbital soft tissue swelling extending into the antrum and ethmoids. (Fig. 4).

An urgent right frontoethmoidectomy, right intranasal antrostomy and drainage of the orbital abscess was performed. The patient was discharged 10 days later but recovered no visual function in the right eye.

\section{DISCUSSION}

The term orbital cellulitis encompasses a variety of acute bacterial inflammatory processes which range in the extent of involvement of the orbit and adnexae. The degree of orbital sepsis was categorized by Chandler et al. (1970) (Table 1). The signs according to progessive severity are illustrated in Table 2.

This classification is an important indicator of mortality and morbidity. In a reported series complication rates from orbital cellulitis ranges from $4-26 \%$ (Jackson \& Baker, 1986). Complications include abscess formation, permanent blindness, limitation of movement of the globe, diplopia, cavernous sinus thrombosis, brain abscess and meningitis.

Peri-orbital soft tissue bacterial infections are relatively common during childhood probably resulting from minor trauma to the eyelid. By contrast, orbital cellulitis is rare and associated frequently with serious morbidity (Spires \& Smith, 1986). Orbital cellulitis is most frequently attributed to acute sinusitis (Chandler et al., 1970), in particular, involvement of the ethmoid and maxillary sinuses has been cited (Mills \& Kartush, 1985). The infection spreads in two ways, direct extension

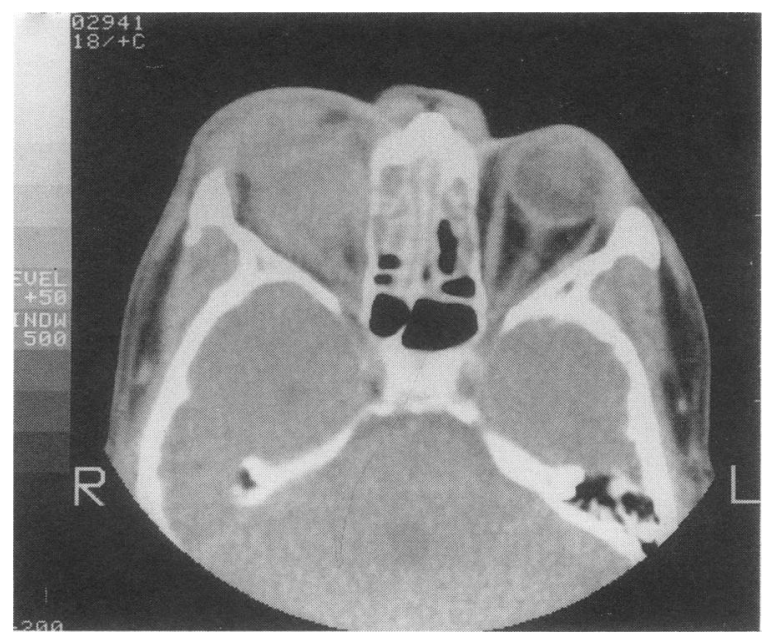

Fig. 4. C.T. scan showing extensive enhancing peri-orbital soft tissue swelling, extending into the antrum and ethmoids. 
Table 1. Classification of orbital cellulitis.

\begin{tabular}{lc}
\hline Category & Group \\
\hline Inflammatory oedema & $1^{*}$ \\
(preseptal cellulitis) & \\
Orbital cellulitis & 2 \\
Subperiosteal abscess & 3 \\
Orbital abscess & 4 \\
Cavernous sinus thrombosis & 5 \\
\hline${ }^{*}$ Chandler's Grouping &
\end{tabular}

Table 2. Clinical signs with progressive severity.

\begin{tabular}{l} 
Signs \\
\hline Pyrexia, unilateral eyelid erythema and oedema, \\
Conjunctivitis, chemosis \\
Orbital tenderness \\
Pain with eye movement \\
Opthalmoplegia \\
Impairement of visual acuity \\
Proptosis \\
Prostration \\
Contralateral signs \\
Greisinger's sign \\
Menigism \\
Death
\end{tabular}

and retrograde thrombophlebitis/thromboembolism along the valveless facial veins (Chandler et al., 1970).

All patients with acute orbital infections should have a comprehensive ophthalmic, ENT and neurological examination. Blood should be drawn for full blood count and culture. Specimens from the abscess and sinus aspirates should be taken for cultures. Sinus radiology is essential to assess the degree and location of the inflammatory process. If any degree of displacement of the globe, ophthalmoplegia or visual impairment is present an urgent C.T. scan is mandatory.

Infection is rarely due to single organisms and Streptococcus, Staphylococcus, Bacteriodes, Haemophilus and anaeorbic Streptococcus are commonly found (Spires \& Smith, 1986). Intravenous broad-spectrum antibiotics should be started immediately and tailored to culture results.

Serial assessment of the patient's visual acuity and occular motility should be performed. The presence of C.T. evidence of intracranial involvement, a subperiosteal or orbital abscess, failure to respond to medical therapy, decreasing vision and worsening ocular motility are indications for immediate exploration and decompression of the orbit. 


\section{ACKNOWLEDGEMENT}

We thank Mr W. T. Farrington, for permission to report these cases and his kind help in preparing the manuscript.

\section{REFERENCES}

Chandler, J. R., Landenbrunner, D. J. \& Stevens, E. R. (1970) The pathogenesis of orbital complications in acute sinusitis. Laryngoscope 80, 1414-1428.

Jackson, K. \& Baker, S. R. (1986) Clinical implications of orbital cellulitis. Laryngoscope 96, 568-574.

Mills, R. P. \& Kartush, J. M. (1985) Orbital wall thickness and the spread of infection from the paranasal sinuses. Clinical Otolaryngology 10, 209-216.

Spires, J. R. \& Smith, R. J. H. (1986) Bacterial infections of the orbital and periorbital soft-tissues in children. Laryngoscope 96, 763-767. 